7th International Symposium on Superalloy 718 and Derivatives Edited by: E.A. Ott, J.R. Groh, A. Banik, I. Dempster, T.P. Gabb, R. Helmink, X. Liu, A. Mitchell, G.P. Sjöberg, and A. Wusatowska-Sarnek TMS (The Minerals, Metals \& Materials Society), 2010

\title{
ATOMIC-LEVEL CHARACTERIZATION OF GRAIN-BOUNDARY SEGREGATION AND ELEMENTAL SITE-LOCATION IN NI-BASE SUPERALLOY BY ABERRATION-CORRECTED SCANNING TRANSMISSION ELECTRON MICROSCOPY
}

\author{
Masashi Watanabe ${ }^{1}$ \\ ${ }^{1}$ Dept. of Mater. Sci. \& Eng., Lehigh University, Bethlehem, PA 18015, USA.
}

Key words: X-ray energy dispersive spectrometry, Electron energy-loss spectrometry, spectrum imaging, Multivariate statistical analysis, Atomic-resolution chemical mapping

Chemical analysis at atomic-level spatial resolution with single-atom detection sensitivity is one of the ultimate goals in materials characterization. Such atomic-level materials characterization would be possible by electron energy-loss spectrometry (EELS) and X-ray energy dispersive spectrometry (XEDS) in aberration-corrected scanning transmission electron microscopes (STEMs) because more probe current can be added into the incident probe by aberrationcorrection. Using aberration-corrected STEMs, image resolution has already reached subÅngstrom levels in high-angle annular dark-field (HAADF) imaging [e.g. 1]. For EELS analysis, sufficient amounts of core-loss signals can be generated within a short acquisition time by higher current probes, and hence atomic-resolution EELS mapping has already been applied [e.g., 2-4]. For XEDS analysis, spatial resolution reaches $\sim 0.4 \mathrm{~nm}$ [5], which implies atomic-level analysis is feasible, in aberration-corrected STEMs. However, atomic-level chemical analysis is even more challenging in the XEDS approach since detection of X-ray signals is more limited than that in EELS ( $\sim 100$ times difference). The limited signals can be improved by applying spectrum-imaging (which records a full spectrum at individual pixels) in combination with multivariate statistical analysis (MSA) [6].

At Lehigh University, two types of aberration-corrected instruments are available: a JEOL JEM$2200 \mathrm{FS}(200 \mathrm{kV}) \mathrm{STEM} / \mathrm{TEM}$ integrated with a CEOS aberration-corrector system and an incolumn $\Omega$ energy-filter [7], and the VG HB 603 (300 kV) dedicated STEM with a Nion aberration-corrector system and an Oxford INCA Si(Li) XEDS system [5]. The former instrument has been optimized for EELS analysis in the atomic-resolution HAADF-STEM imaging mode. The latter is being used primarily for high-resolution XEDS analysis. In this study, both the aberration-corrected instruments were applied to the characterization of a Ni-base superalloy $\mathrm{X}-750$, which contains $\mathrm{L1}_{2}$-ordered $\gamma^{\prime}$ precipitates in a $\gamma$ matrix.

Figure 1 shows an atomic-resolution HAADF-STEM image recorded from a [001]-projected $\gamma / \gamma^{\prime}$ interface by the JEM-2200FS. The primary XEDS analysis shows that the Ti composition in atomic $\%$ is twice as high as $\mathrm{Al}$ in the $\gamma^{\prime}$ precipitates [5]. However, the precipitates still maintain the $\mathrm{Ni}_{3} \mathrm{Al}$-type $\mathrm{L}_{2}$ structure rather than the $\mathrm{Ni}_{3}$ Ti-type $\mathrm{DO}_{24}$ structure. In the [001] projection of the alloy, the atomic spacing is $\sim 1.8 \AA$, which is clearly resolved in the HAADF-STEM image. From the HAADF-STEM image, the $\gamma$ ' precipitates have a coherent interface with the matrix $\gamma$ phase. Additionally, two types of major spots can be seen in the $\gamma$ precipitate region of the image. The brighter and darker spots correspond to face-centered (A site) and corner (B site) atom positions in the $\mathrm{L}_{2}$ structure, respectively. Although the contrast corresponding to the 
atomic-column positions clearly changes, no local shift in atomic configuration was observed across the $\gamma / \gamma^{\prime}$ interface. Figure 2 shows two EELS spectra around the Ti $\mathrm{L}_{2,3}$ edge $(\sim 450 \mathrm{eV})$ recorded from the A (a) and B (b) sites, respectively [7]. It is clear that the $\mathrm{Ti} \mathrm{L}_{2,3}$-edge intensity is much higher in the spectrum from the $\mathrm{B}$ site. The difference in the $\mathrm{Ti} \mathrm{L}_{2,3}$-edge signals between A and B sites suggests that the Ti atoms occupy preferentially the B-site, which agrees with previous studies using the ALCHEMI (atom location by channeling-enhanced microanalysis) method via X-rays [8]. Therefore, using aberration-corrected STEMs, such site occupancies of alloying elements or even impurity elements can be determined directly at the individual atomic-column level, not by averaging information from broad regions as traditionally carried out using ALCHEMI.

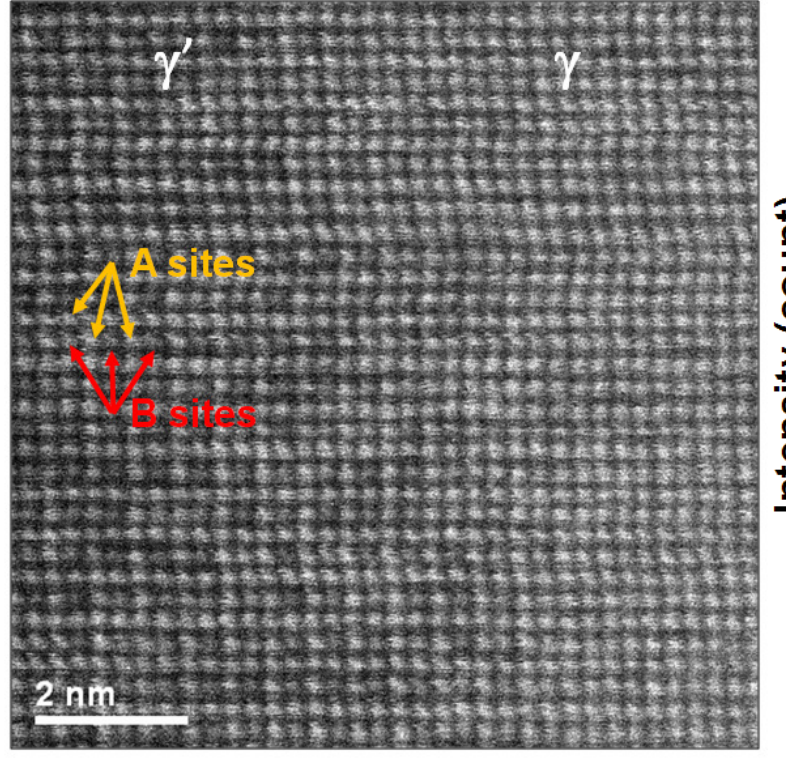

Fig. 1: A high-resolution HAADFSTEM image taken from a $\gamma / \gamma^{\prime}$ interface by the JEM-2200FS in the STEM mode.

(a) Fe $\mathrm{L}_{2,3} \quad \gamma^{\prime}$

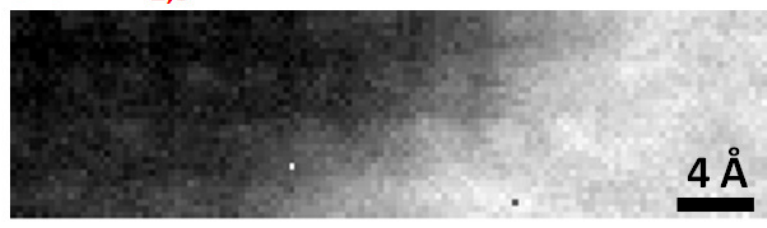

(b) $\mathrm{Ti}_{2,3}$

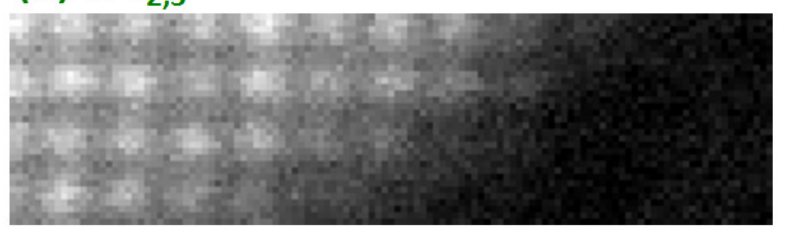

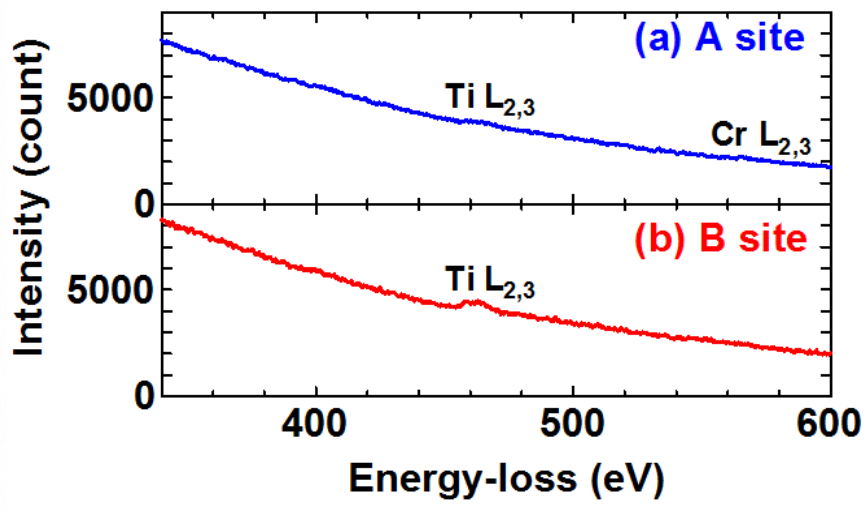

Fig. 2: EELS spectra taken from the A (a) and B (b) site positions in the $\gamma$ ' phase region by JEM-2200FS in the STEM mode [7].

(c) Ni $\mathrm{L}_{2,3}$

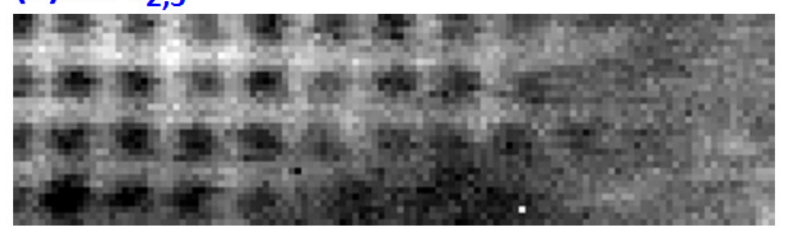

(d) RGB overlay

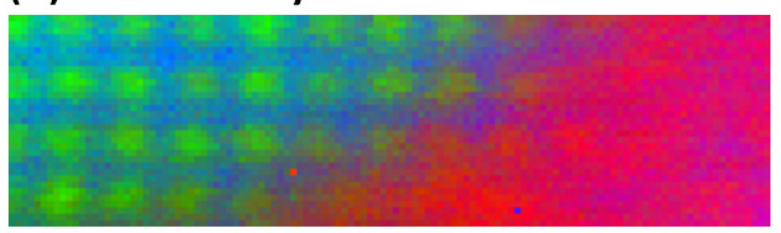


Furthermore, the same specimen was analyzed by using the recently developed aberrationcorrected STEM JEOL JEM-ARM200F (which is fully optimized for atomic-resolution chemical analysis [9]) at JEOL factory in Japan. Figure 3 shows elemental maps of (a) $\mathrm{Fe} \mathrm{L}_{2,3}$, (b) $\mathrm{Ti} \mathrm{L}_{2,3}$ and (c) $\mathrm{Ni} \mathrm{L}_{2,3}$ obtained from the vicinity of $\gamma / \gamma^{\prime}$ interface in [001] projection. The RGB color overlay image is also shown in Fig. 3(d) to see elemental distribution more clearly. In the $\mathrm{L}_{12^{-}}$ type ordered $\gamma^{\prime}$ phase, Ti preferentially occupies corner sites as shown in Fig. 2. The Ti map shows that $\mathrm{Ti}$ is mainly distributed in the $\gamma^{\prime}$ phase, specifically at the corner sub-lattices. Conversely, Ni signals are depleted at the corner sub-lattices in the $\gamma^{\prime}$ phase. In this experiment, the spatial resolution was not high enough to resolve Ni atomic columns in the $\gamma$ phase, which correspond to $1.8 \AA$, because a higher probe current to generate sufficiently enough EELS signals for mapping degrades the resolution. However, it is encouraging to perform atomicresolution characterization by using the latest instrument.

Spatial resolution and analytical sensitivity of X-ray analysis should also be improved by using an aberration-corrected STEM. Figure 4 (a) shows an HAADF-STEM image around a grain boundary (GB) in the same Ni-base alloy. There is a coarse $\gamma^{\prime}$ precipitate at the top right in this area along the GB, and hence the GB is an interphase interface between the $\gamma / \gamma$ ' phases at the topleft corner. Then this GB becomes an ordinary $\gamma / \gamma$ at the lower region. An X-ray spectrumimaging dataset was acquired from the square region at the GB in the aberration-corrected VG HB 603 and analyzed by the MSA method. In Fig. 4 (b), an interesting component extracted from the dataset by MSA is shown, including the loading spectrum and corresponding score image. The loading spectrum shows positive $\mathrm{K}$ and $\mathrm{L}$ peaks of $\mathrm{Zr}$ and $\mathrm{Nb}$ and negative $\mathrm{K}$ peaks of $\mathrm{Ti}, \mathrm{Cr}$ and $\mathrm{Ni}$. It should be noted that the positive signals in the loading spectrum are enhanced and the negatives are reduced from the main information. This data enhancement occurs at brighter regions in the corresponding score image. In this case, the brighter region in the score image is superimposed on the GB. Thus both $\mathrm{Zr}$ and $\mathrm{Nb}$ are enriched (i.e., cosegregated) at the GB.

The composition map of $\mathrm{Zr}$ quantified after the MSA noise reduction are shown in Fig. 5 (a). $\mathrm{Zr}$ is present at a nominal $0.04 \mathrm{wt} \%$ (well below the detection limit!), so it has not been quantified or mapped previously in this alloy via X-ray analysis. To the authors' knowledge, there is no report of GB segregation of either $\mathrm{Zr}$ or $\mathrm{Nb}$ in this alloy. A concentration line profile extracted from the $\mathrm{Zr}$ map across the $\gamma / \gamma^{\prime}$ interface region is shown in Fig. 5 (b). To improve counting statistics, the profile was constructed by binning 20 pixels along the interface. The error bar represents a $99 \%$ confidence limit $(3 \sigma)$. The error levels are relatively high due to the shorter acquisition time and much lower composition of the alloying elements, but the $\mathrm{Zr}$ composition at the GB can still be clearly distinguished from the $\mathrm{Zr}$ composition within the grains. The spatial resolution determined from these segregation profiles is as good as 0.4-0.6 nm at FWTM, which is the best spatial resolution that has ever been obtained in X-ray analysis. Maps of the $\mathrm{Zr}$ enrichment and the number of $\mathrm{Zr}$ atoms calculated by the $\zeta$-factor method [10] are shown in Fig. 5(c) and (d), respectively. The detailed approach for extracting this information can be found in previous papers $[10,11]$. The $\mathrm{Zr}$ enrichment on the GB is $2-3$ atoms $/ \mathrm{nm}^{2}$, as obtained from the enrichment map, which corresponds to $0.12-0.17$ monolayer. It should be noted that a single monolayer is assumed as a close-packed (111) plane in this ally, which is equivalent to $\sim 17$ atoms/nm2. As shown in Fig. 5(d), enrichment amounts are only 20-40 Zr atoms at the GB. Such limited amounts of enriched atoms (which are highly localized in limited regions) may simply be invisible to the relatively larger probe in the conventional uncorrected condition. Therefore, not only spatial resolution but also analytical sensitivity is significantly improved in the aberrationcorrected STEMs. 
(a)

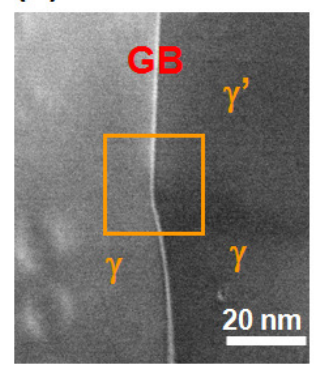

(b) Loading (decomposed feature)

Score (amplitude)

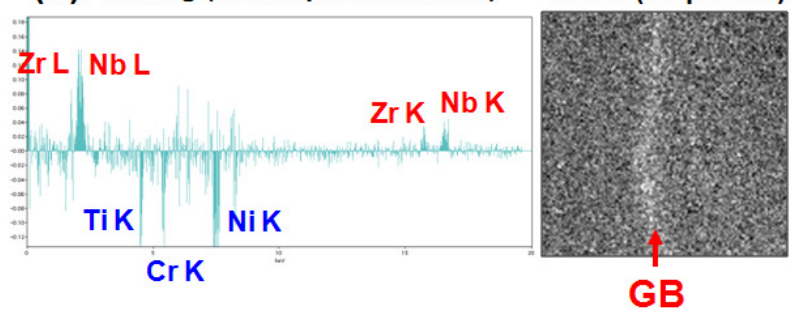

Fig. 4: (a) an HAADF-STEM image taken around $\mathrm{a} G \mathrm{~GB}$ in a Ni-based alloy. An X-ray SI dataset was acquired from the selected squared region in aberration-corrected VG HB 603 STEM and (b) a pair of loading spectrum and the score image of a statistically significant component extracted from the X-ray SI dataset by applying MSA [5]. (a)

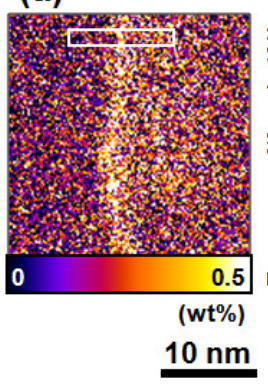

(c)

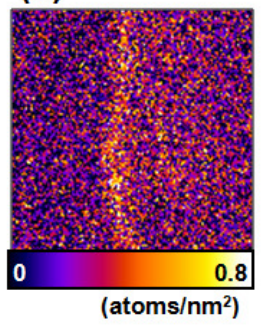

(b)

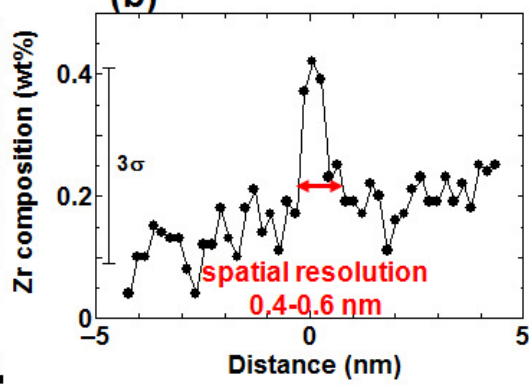

(d)

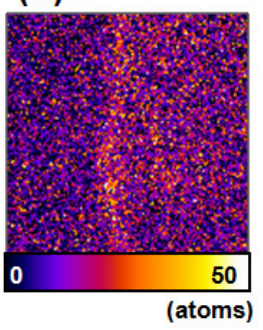

Fig. 5: (a) $\mathrm{Zr}$ composition map quantified by the $\zeta$-factor method after the MSA noise-reduction. (b) A $\mathrm{Zr}$ composition profile across the $\gamma / \gamma^{\prime}$ interface on the GB. The error bar indicates a $99 \%$ confidence level $(3 \sigma)$ and the spatial resolution determined from the profiles is $\sim 0.4-0.6 \mathrm{~nm}$ (FWTM). Maps of Zr enrichment (c) and the number of $\mathrm{Zr}$ atoms (d) [5].

In this manuscript, atomic-level characterizations of a Ni-base superalloy X-750 by using aberration-corrected STEMs were reviewed as a model case. By EELS analysis on the $\gamma^{\prime}$ phase, the Ti signal is mainly localized at the $\mathrm{Al}$ site, but not at the Ni site, which confirms that $\mathrm{Ti}$ atoms occupy preferentially the Al-site. Furthermore, elemental distributions can be mapped out in atomic-column resolution by the latest aberration-corrected instrument. X-ray analysis can also be improved by the aberration correction in terms of spatial resolution and analytical sensitivity. With the improved spatial resolution and analytical sensitivity, $\mathrm{Zr}$ and $\mathrm{Nb}$ cosegregation was found first time at a GB with a very small composition variation (below $0.5 \mathrm{wt} \%$ between matrix and GB) by MSA data enhancement. Chemical analysis in atomic scale can now, at last, be achieved using the aberration-corrected STEMs.

\section{Acknowledgements}

The author also wishes to acknowledge the support of the National Science Foundation (grant DMR-0804528) and Bechtel Bettis, Inc. The author would like to thank Prof. David Williams (currently at the Univ. of Alabama, Huntsville) for his thoughtful supervision during the development of the MSA plug-ins, and gratitude is extended to Dr. Eiji Okunishi (JEOL Ltd.) for performing atomic-resolution EELS analysis. 


\section{References}

1. P.D. Nellist, et al. Science 305 (2004) 1741.

2. M. Bosman, et al., Phys. Rev. Lett., 99 (2007), 086102.

3. D.A. Muller, et al., Science, 319 (2008), 1073.

4. M. Varela, et al., Phys. Rev. B, 79 (2009), 085117.

5. M. Watanabe, et al., Microsc. Microanal. 12 (2006), 515.

6. M. Watanabe et al., Microsc. Anal., 23 (2009), Issue 7, 5.

7. M. Watanabe et al., JEOL News, 41 (2006), 1.

8. Z. Horita et al. Phil. Mag. A 67 (1993), 425.

9. I. Ishikawa, et al., Microsc. Microanal., 15 (2009), Suppl. 2, 188.

10. M. Watanabe and D.B. Williams, J. Microsc., 221, (2006), 89.

11. M. Watanabe and D.B. Williams, Z. Metallk, 94 (2003), 307. 